\title{
Klotho and Renal Fibrosis
}

\author{
Sepide Zununi Vahed ${ }^{1,2}$, Parisa Nikasa ${ }^{1,2}$, Mohammadreza Ardalan ${ }^{2, *}$ \\ ${ }^{1}$ Faculty of Advanced Medical Sciences, Tabriz University of Medical Sciences, Tabriz, IR Iran \\ ${ }^{2}$ Chronic Kidney Disease Research Center, Tabriz University of Medical Sciences, Tabriz, IR Iran \\ *Corresponding author: Mohammadreza Ardalan, Chronic Kidney Disease Research Center, Tabriz University of Medical Sciences, Tabriz, IR Iran. Tel: +98-9141168518, Fax: +98- \\ 4113366579, E-mail: ardalan34@yahoo.com.
}

Received: November 5, 2013; Accepted: November 11, 2013

Keywords: Klotho Protein; Nephrogenic Fibrosing Dermopathy; Renal Insufficiency, Chronic; Fibrosis

Renal fibrosis is a pathological condition that characterized by excessive accumulation of extracellular matrix (ECM) and it is a common pathway of progression in different renal diseases including chronic glomerulonephritis, diabetic nephropathy chronic allograft nephropathy and renal senescence $(1,2)$. In the process of renal fibrosis leukocyte infiltration, activation of fibroblasts and myofibroblasts, epithelial-mesenchymal transition (EMT) all have been identified as the major events (3). Behind The pathological features there are complex orchestrated interplay of different factors including cytokines (TGF- $\beta$, IFN- $\gamma$, IL-10), transcriptional activator factors (Sp1, Egr-1, Smad3), cell surface repressors down regulation or up regulation (Smad7, Fli-1, PPAR- $\gamma$, p53, Klotho) and epigenetic modulators (acetyltransferase, methyltransferases, deacetylases, microRNAs) (4). Deregulation of pro-fibrotic and down regulation of anti-fibrotic factors play an important role in initiation and progression of renal fibrosis. Understanding the mechanism would be valuable to diagnose, prevent and even reverse the process of fibrosis. The role of micro RNA as a biomarker or as a therapeutic modality of renal fibrosis has recently be discussed by authors (5).

Klotho, was first identified as an anti-aging protein in 1997, and it spawned two decades of aging research (6). Klotho is expressed in different tissues including kidney, heart, brain, and parathyroid but it is particularly highly expressed in kidney and predominantly in distal and proximal convoluted tubules. Klotho is a single-pass transmembrane (130 KDa) protein $(6,7)$. Membrane-anchored Klotho acts as a co-receptor for fibroblast growth factor-23 that promote renal phosphate excretion and suppresses active vitamin D synthesis $(8,9)$ soluble Klotho $(70 \mathrm{kD})$ is generated from the Klotho gene through alternative splicing. It could also sheds from cell mem- brane (9). Soluble Klotho (sKl) acts as a hormonal with multiple renal and extrarenal functions including suppression of fibrosis (10), antioxidant activity (11), preservation of stem cells (12), down regulation of Wnt signaling and acting as an anti-aging agent (13) and modulation of ion transport (14). Klotho-deficient mice develop severe kidney damage and fibrosis. $\mathrm{Kl}-1$ - deficient mice with unilateral ureteral obstruction (UUO) had higher TGF- $\beta 1$ levels and more renal fibrosis than WT wild type mice with UUO $(13,15)$ in human kidney, decreased Klotho expression occurs in early stages of CKD, and could be responsible for early increase in serum FGF23 level (16). Klotho deficient animals develops accelerated vascular calcification (17). Cell culture studies have confirmed that Klotho deficiency directly promotes renal epithelial cell senescence $(14,18,19)$.

Experimental studies have revealed that Klotho level decreases in acute and chronic kidney diseases (12) and Klotho has a nephron-protective role $(6,9,10,12,20)$. Klotho acts as an endogenous inhibitor of multiple growth factor including transforming growth factor- $\beta 1$ (TGF-ß1), Wnt, and IGF-1 $(8,10,12)$. Klotho primarily suppresses renal fibrosis through inhibition of TGF-ß1 signaling (21), and plays a central role in the pathogenesis of renal fibrosis in both experimental and human kidney diseases $(22,23)$. TGF- $\beta 1$ induces matrix production and inhibits ECM degradation via suppression of matrix metalloproteinases (MMPs) and induction of the of MMPs inhibitors (TIMPs) $(4,16)$. TGF- $\beta 1$ inhibits expression of genes that are essential for epithelial phenotype such as E-cadherin, and induces expression of factors that are essential for mesenchymal phenotype such as vimentin, collagen-1, and N-cadherin respectively (17). Secreted Klotho hinders profibrotic effects of TGF- $\beta 1$ through direct interaction with TGF- $\beta$ receptor II (8). TGF- $\beta 1$ itself as a new therapeutic strategy to combat the renal fibrosis.

Copyright (c) 2013, Nephrology and Urology Research Center; Published by Kowsar Corp. This is an open-access article distributed under the terms of the Creative Commons Attribution License, which permits unrestricted use, distribution, and reproduction in any medium, provided the original work is properly cited. 
could suppress the tissue expression of Klotho $(10,12,18)$. Klotho is a critical negative regulator of Wnt signaling that not only acting as an anti-aging agent (13), but also it has a critical role in normal wound healing, and its sustained activation is associated with fibrogenesis $(19,24)$. Klotho binds to Wnt ligands and represses Wnt-induced transcription of $\beta$-catenin targets in response to TGF- $\beta 1$. Invivo expression of secreted Klotho in inhibits the activation of renal $\beta$-catenin, and decreases ECM deposition (12). These results suggest that Klotho is an endogenous antagonist of Wnt and $\beta$-catenin activity that inhibits the activation of renal myofibroblast, EMT and renal fibrosis (12). Senescent cells secrete altered levels of growth factors that future increase susceptibility to apoptosis, and delay the repair and regeneration in the aging kidney (5, 23). From histological point of view, renal senescence is characterized by reduction in cortical mass, glomerulosclerosis, interstitial fibrosis/tubular atrophy (IF/TA) and arteriosclerosis $(21,25)$. Telomere shortening and cell cycle arrest are physiologic contributors of aging and from pathophysiologic causes; oxidative stress, epigenetic alterations, mitochondrial injury and immunosuppressive agent are important contributors $(2,25,26)$. Soluble klotho binds to Wnt protein and inhibits cell senescence through the inhibition of Wnt signaling (13). Maekawa et al. showed the anti-senescence properties of exogenous administrated Klotho (27). Therefore, it could be considered a therapeutic modality for prevention of IF/TA in renal allograft.

Increased activity of plasminogen activator inhibitor 1 (PAI-1) is associated with kidney fibrosis it can be seen in chronic allograft nephropathy, chronic glomerulonephritis, diabetic nephropathy, and hypertensive nephrosclerosis $(28,29)$. PAI-1 mRNA and PAI-1 protein activity were strikingly elevated in multiple tissues of Klothodeficient $\left(\mathrm{Kl}^{-{ }^{-}}\right)$mice (9), and Klotho supplementation might reduce PAI-1 activity (9). Sugiura et al. (2010) reported that $\mathrm{Kl}^{-1}-$ deficient mice had higher levels of renal tubulointerstitial fibrosis that was associated with upregulation of TGF- $\beta 1$ (30).

Klotho supplementation inhibits renal fibrosis by suppression of fibrotic markers includings; $\alpha$-smooth muscle actin, Fibronectin, Vimentin, collagen-1, MMPs. and TGF- $\beta 1$ (8). Soluble Klotho can suppress renal fibrosis and preserve renal function in UUO model of renal injury, therefore it could be considered as a novel therapeutic agent in renal fibrosis (8). Chronic kidney disease (CKD) and aging going hand in hand. Indoxyl sulfate is a uremic toxin that reduces renal Klotho expression, and contributes to cell senescence and renal fibrosis $(30,31)$.

Increased methylation of Klotho promoter, microRNA-339 and microRNA-556 could directly decrease Klotho expression in cultured cells (32). Klotho promotes kidney regeneration after ischemia-reperfusion injury via the suppression of fibrosis-promoting growth factors, pres- ervation of stem cells, and the recovery of endothelial integrity and function, (33) Klotho incubation mitigates cell senescence and apoptosis in endothelial cells triggered by oxidative stress or by Klotho deficiency $(10,19)$. These properties could be considered as therapeutic measurements in future.

Klotho opens a new venue in renal fibrosis research. it is a promising diagnostic marker, a prognostic marker and a therapeutic agent $(9,20)$. Preventing its down regulation by targeting the factors that decrease its expression, upregulation of its endogenous production or its external repletion would be applicable to develop new therapeutic strategies for renal fibrosis.

\section{Financial Disclosure}

There is no financial disclosure.

\section{References}

1. Conway B, Hughes J. Cellular orchestrators of renal fibrosis. QJM. 2012;105(7):611-5.

2. Yang H, Fogo Agnes B. Cell senescence in the aging kidney. J Am Soc Nephrol. 2010;21(9):1436-9.

3. Carew RM, Wang B, Kantharidis P. The role of EMT in renal fibrosis. Cell Tissue Res. 2012;347(1):103-16.

4. Ghosh Asish K, Quaggin Susan E, Vaughan Douglas E. Molecular basis of organ fibrosis: Potential therapeutic approaches. Experiment Biol Med. 2013;238(5):461-81.

5. Zununi S, Ardalan M. MicroRNA and Renal Allograft Monitoring. 2013;5(3):783-6.

6. Hu MC, Kuro-o M, Moe OW, editors. Renal and Extrarenal Actions of Klotho; Seminars in nephrology; 2013; Elsevier; pp. 118-129.

7. Kuro-o M, Matsumura Y, Aizawa H, Kawaguchi H, Suga T, Utsugi T, et al. Mutation of the mouse klotho gene leads to a syndrome resembling ageing. Nature.1997;390(6655):45-51.

8. Doi S, Zou Y, Togao O, Pastor JV, John GB, Wang L, et al. Klotho inhibits transforming growth factor- $\beta 1$ (TGF- $\beta 1$ ) signaling and suppresses renal fibrosis and cancer metastasis in mice. J Biol Chemist. 2011;286(10):8655-65.

9. Hu M, Moe OW. Klotho as a potential biomarker and therapy for acute kidney injury. Nature Rev Nephrol. 2012;8(7):423-9.

10. Sugiura H, Yoshida T, Shiohira S, Kohei J, Mitobe M, Kurosu H, et al. Reduced Klotho expression level in kidney aggravates renal interstitial fibrosis. Am J Physiol Renal Physiol. 2012;302(10):F1252-64.

11. Kuro-o M. Klotho as a regulator of oxidative stress and senescence. Biol chem. 2008;389(3):233-41.

12. Zhou L, Li Y, Zhou D, Tan RJ, Liu Y. Loss of Klotho Contributes to Kidney Injury by Derepression of Wnt/ $\beta$-Catenin Signaling. J Am Soc Nephrol. 2013;24(5):771-85.

13. Liu H, Fergusson MM, Castilho RM, Liu J, Cao L, Chen J, et al. Augmented Wnt signaling in a mammalian model of accelerated aging. Science. 2007;317(5839):803-6.

14. Cha S, Hu M, Kurosu H, Kuro-o M, Moe O, Huang C. Regulation of renal outer medullary potassium channel and renal $\mathrm{K}+$ excretion by Klotho. Molecul pharmacol. 2009;76(1):38-46.

15. Caigan Du. Transforming Growth Factor-Beta in Kidney Transplantation: A Double-Edged Sword. In: Trzcinska ML, editor. Kidney Transplantation - New Perspectives. pp. 223-34.

16. Wynn TA. Cellular and molecular mechanisms of fibrosis. $J$ Pathol. 2008;214(2):199-210.

17. Zeisberg M, Neilson EG. Biomarkers for epithelial-mesenchymal transitions. J Clin Investigat. 2009;119(6):1429.

18. Hu MC, Kuro-o M, Moe OW. Secreted klotho and chronic kidney disease. Endocrine FGFs and Klothos. Springer; 2012. pp. 126-157.

19. Satoh Minoru, Nagasu Hajime, Morita Yoshitaka, Yamaguchi Terry P, Kanwar Yashpal S, Kashihara Naoki. Klotho protects against 
mouse renal fibrosis by inhibiting Wnt signaling. Am J Physiol Renal Physiol. 2012;303(12):F1641-51.

20. Hu MC, Kuro-o M, Moe OW. The emerging role of Klotho in clinical nephrology. Nephrol Dial Transplant. 2012;27(7):2650-7.

21. Iglesias-De La Cruz C, Ruiz-Torres P, del Moral RG, Rodríguez-Puyol M, Rodríguez-Puyol D. Age-related progressive renal fibrosis in rats and its prevention with ACE inhibitors and taurine. Am J Physiol Renal Physiol. 2000;278(1):F122-9.

22. Farris AB, Colvin RB. Renal Interstitial Fibrosis: Mechanisms and Evaluation In: Current Opinion in Nephrology and Hypertension. Cur Opinion Nephrol Hypertens. 2012;21(3):289-300.

23. Caigan Du. Transforming Growth Factor-Beta in Kidney Transplantation: A Double-Edged Sword. In: Trzcinska ML, editor. Kidney Transplantation - New Perspectives. pp. 223-34.

24. Lam Anna P, Gottardi Cara J. $\beta$-catenin signaling: a novel mediator of fibrosis and potential therapeutic target. Cur Opinion Rheumatol. 2011;23(6):562-7.

25. Braun H, Schmidt BM, Raiss M, Baisantry A, Mircea-Constantin D, Wang S, et al. Cellular senescence limits regenerative capacity and allograft survival. J Am Soc Nephrol. 2012;23:1467-73.

26. Jennings P, Koppelstaetter C, Aydin S, Abberger T, Wolf AM, Mayer $G$, et al. Cyclosporine A induces senescence in renal tubular epithelial cells. Am J Physiol Renal Physiol. 2007;293(3):F831-8.
27. Maekawa Y, Ohishi M, Ikushima M, Yamamoto K, Yasuda O, Oguro $\mathrm{R}$, et al. Klotho protein diminishes endothelial apoptosis and senescence via a mitogen activated kinase pathway. Geriatric Gerontol Int. 2011;11(4):510-6.

28. Samarakoon R, Overstreet JM, Higgins SP, Higgins PJ. TGF- $\beta 1 \rightarrow$ $\mathrm{SMAD} / \mathrm{p} 53 / \mathrm{USF} 2 \rightarrow \mathrm{PAI}-1$ transcriptional axis in ureteral obstruc tion-induced renal fibrosis. Cell Tissue Res. 2012;347(1):117-28.

29. Eddy Allison A, Fogo Agnes B. Plasminogen activator inhibitor-1 in chronic kidney disease: evidence and mechanisms of action. $J$ Am Soc Nephrol. 2006;17(11):2999-3012.

30. Sugiura H, Yoshida T, Kohei J. TGF- $\beta$ was upregulated in renal fibrosis model of Klotho defect mouse and affected renal Klotho expression level.J Am Soc Nephrol. 2010;21:376A.

31. Barreto FC, Barreto DV, Liabeuf S, Meert N, Glorieux G, Temmar $\mathrm{M}$, et al. Serum indoxyl sulfate is associated with vascular disease and mortality in chronic kidney disease patients. Clin J Am Soc Nephrol. 2009;4(10):1551-8.

32. Mehi Stephen J, Maltare A, Abraham Carmela R, King Gwendalyn D. MicroRNA-339 and microRNA-556 regulate Klotho expression in vitro. AGE. 2013:[Epub ahead of print].

33. Sugiura H, Yoshida T, Shiohira S, Kohei J, Mitobe M, Kurosu H, et al. Reduced Klotho expression level in kidney aggravates renal interstitial fibrosis. Am J Physiol Renal Physiol. 2012;302(10):F1252-64. 